\title{
BMJ Open Implementation of the Symptom Navi (C) Programme for cancer patients in the Swiss outpatient setting: a study protocol for a cluster randomised pilot study (Symptom Navi@ Pilot Study)
}

To cite: Bana M, Ribi K, KropfStaub S, et al. Implementation of the Symptom Navi (C) Programme for cancer patients in the Swiss outpatient setting: a study protocol for a cluster randomised pilot study (Symptom NaviC) Pilot Study). BMJ Open 2019;9:e027942. doi:10.1136/ bmjopen-2018-027942

- Prepublication history and additional material for this paper are available online. To view these files, please visit the journal online (http://dx.doi org/10.1136/bmjopen-2018027942).

Received 15 November 2018 Revised 2 May 2019 Accepted 9 May 2019

Check for updates

(C) Author(s) (or their employer(s)) 2019. Re-use permitted under CC BY-NC. No commercial re-use. See rights and permissions. Published by BMJ.

For numbered affiliations see end of article.

Correspondence to

Dr Manuela Eicher;

manuela.eicher@chuv.ch

\section{ABSTRACT}

Introduction Self-management interventions show promising results on symptom outcomes and selfmanagement behaviours. The Symptom Navi@ Programme (SNOP) is a nurse-led intervention supporting patients' symptom self-management during anticancer treatment. It consists of written patient information (Symptom Navi@ Flyers (SNCFlyers)), semistructured consultations and a training manual for nurses.

Methods and analysis This pilot study will evaluate the implementation of the SNCP based on the Reach Effectiveness-Adoption Implementation Maintenance framework at Swiss outpatient cancer centres. We will use a cluster-randomised design and randomise the nine participating centres to the intervention or usual care group. We expect to include 140 adult cancer patients receiving first-line systemic anticancer treatment. Trained nurses at the intervention clusters will provide at least two semistructured consultations with the involvement of SNCFlyers. Outcomes include patients' accrual and retention rates, patient-reported interference of symptoms with daily functions, symptom burden, perceived self-efficacy, quality of nursing care, nurse-reported facilitators and barriers of adopting the programme, nurses' fidelity of providing the intervention as intended, and patients' safety (patients timely reporting of severe symptoms). We will use validated questionnaires for patient-reported outcomes, focus group interviews with nurses and individual interviews with oncologists. Linear mixed models will be used to analyse patient-reported outcomes. Focus group and individual interviews will be analysed by thematic analysis.

Ethics and dissemination The Symptom Navi@ Pilot Study has been reviewed and approved by Swiss Ethic Committee Bern (KEK-BE: 2017-00020). Results of the study will be disseminated in peer-reviewed journal and at scientific conferences.

Trial registration number NCT03649984; Pre-results.

\section{INTRODUCTION}

Anticancer treatments are increasingly provided in the outpatient setting. ${ }^{12}$ Cancer
Strengths and limitations of this study

One strength of the study protocol is its integration in a larger research and development programme: after several steps of development and content validation of the Symptom NaviC Programme (SNCP), we now conduct a pilot implementation study including the evaluation of preliminary effectiveness of the SNCP.

- This pilot study explores the implementation of the SNCP based on the Reach Effectiveness-Adoption Implementation Maintenance framework.

- We apply a cluster-randomised design with nine Swiss outpatient cancer centres allocated to the implementation of the SNCP or usual care complemented with qualitative methods.

- We assess patient-reported outcomes over 16 weeks to explore effect sizes for calculating the sample size for a full powered cluster randomised controlled trial (RCT).

- Long-term impact and maintenance of the SN@P are not included in this pilot study and will need further investigation.

outpatients report substantial symptom burden related to disease and side effects of anticancer treatments. ${ }^{3}$ Symptom intensity usually increases between treatment applications, ${ }^{5}$ when patients are at home and healthcare providers are not immediately available. Cancer patients report unmet supportive care needs to learn how to self-manage their symptoms. $^{6}$

Symptom self-management is a dynamic process of integrating adequate behaviours and strategies to prevent, relieve or decrease symptoms. ${ }^{7}$ This process includes symptom and treatment management, dealing with the emotional and physical consequences of disease and treatment, and adapting 
life roles. ${ }^{8}$ Self-management behaviours are based on several core competencies including problem solving, decision-making, communication with healthcare professionals, tailoring recommendations to the individual situation and taking action. ${ }^{10-12}$ There are two core elements of self-management interventions that are most frequently applied: (1) tailoring the content of the intervention to patient's needs and (2) facilitating patient's self-efficacy by using goal setting and action planning. ${ }^{13}$

Self-efficacy is a subjective belief that a person can achieve a planned task or action, even if it becomes challenging. ${ }^{14}$ Fostering patient self-efficacy is a pivotal core element because of its impact on patient self-management behaviours. ${ }^{1315}$ Self-efficacy is a mediator for a persons' ability to acquire self-management behaviours ${ }^{10} 14$ and to manage symptoms. ${ }^{9} 12$ Therefore, supporting self-efficacy might play a key role for self-management interventions and successful self-management behaviours.

It is still unclear what combination of core elements makes a self-management intervention effective ${ }^{13}$ because the format, content and outcomes of the investigated interventions are very heterogeneous. ${ }^{13}$ 16-19 The heterogeneity of intervention and outcomes preclude meta-analyses in systematic reviews and this has led to mainly narrative syntheses. ${ }^{13}$ 18-21 Frequently reported effects of self-management interventions were decreased symptom intensity or burden (eg, fatigue, depression, anxiety, distress), ${ }^{13} 1718$ 21-24 increased quality of life, ${ }^{13} 172223$ better physical functioning ${ }^{19}$ or performance, ${ }^{18}$ and improved self-efficacy. $^{17 \quad 18 \quad 2021}$ However, two systematic reviews reported ambiguous effects on quality of life $\mathrm{e}^{2024}$ and self-efficacy. ${ }^{24}$ Further research should clarify whether the intervention's content was ineffective, or whether contextual factors (eg, nurses' workload) prevented the intended effects.

Important aspects of interventions supporting self-management remain scarcely investigated. A majority of recently published systematic reviews focused on self-management interventions during survivorship or the rehabilitation phase of cancer patients, ${ }^{161719212224}$ and a minority on interventions during active treatment phase. ${ }^{20} 2325$ Studies rarely included a description of how to support patients in communicating their symptoms and asking for support when needed, ${ }^{26}$ or at what moment they have to make contact the care team if a symptom becomes severe. ${ }^{27}$ Trained healthcare professionals who work collaboratively within a multidisciplinary team should provide support and guidance about care seeking. ${ }^{13}$ In most studies, healthcare professionals other than nurses provided the interventions for supporting self-management. $^{202324}$

Nurses are in close contact with cancer patients and should play a key role in supporting symptom self-management. $^{6} 28 \quad 29$ In a randomised controlled trial, a nurse-led intervention for cancer patients during chemotherapy was associated with decreased patient-reported problems, ${ }^{29}$ and showed reduced symptom intensity/burden, improved self-efficacy and enhanced self-management behaviours. ${ }^{30}$ However, nurse-led interventions supporting symptom self-management are challenging and complex because they require a structured but flexible behaviour of nurses in tailoring the intervention to individual situations. ${ }^{15} 3132$ Implementation of such complex interventions should include a thorough analysis of contextual factors (eg, organisational readiness for change, workload) and take into account the resources needed to apply the intervention. ${ }^{33} 34$

In 2011, nurses from a Swiss hospital initiated the Symptom Navi@ Programme (SN@P) for patients during anticancer treatments to address the need of cancer patients asking for more information about symptom management. ${ }^{356}$ The SN@P has received attention from other Swiss cancer centres who are interested to implement this programme.

\section{Aim and objectives}

The overall aim of this study is to evaluate the implementation of the SN@P at Swiss outpatient cancer centres and to explore its preliminary effectiveness compared with usual care. Implementation of newly developed interventions depend on organisational structures and the collaboration of involved stakeholders. ${ }^{37}$ Therefore, we based the evaluation of the implementation process on the Reach Effectiveness-Adoption Implementation Maintenance (RE-AIM) framework. ${ }^{38-40}$ This five-dimension framework considers outcomes on individual and organisational level. The maintenance dimension cannot be addressed in a pilot study.

Patients' perceived self-efficacy is associated with self-management behaviour, symptom outcomes and daily functioning. ${ }^{41}$ The primary objective is to explore the impact of the SN○P on patients' symptom interference with their daily functions (affective and activity) compared with usual care.

Secondary objectives are to:

1. Assess accrual and retention rates of patients (reach).

2. Investigate the impact of the $\mathrm{SN} \odot \mathrm{P}$ on patient symptom severity and burden, and their self-efficacy (effectiveness).

3. Explore barriers and facilitators (eg, work-related factors, available resources) of adopting the SNOP in the outpatient cancer centres (adoption).

4. Explore nurses' fidelity to the SN@P training manual within daily routines, and estimate needed resources to implement the SN@P (implementation).

5. Explore patients' evaluation on nurses' support for symptom management (implementation).

\section{METHODS AND ANALYSIS}

\section{Design}

We will apply a cluster-randomised design with two parallel arms complemented with qualitative methods. The unit of randomisation is the participating outpatient cancer centre with each centre representing a cluster. A cluster-randomised design was chosen to avoid contamination 
Table 1 Eligibility criteria for patients

\begin{tabular}{ll} 
Inclusion criteria & Exclusion criteria \\
\hline 18years and older. & Insufficiently literate in German. \\
Newly diagnosed with any early or advanced/metastatic & Diagnosed with a recurrence of cancer disease. \\
cancer disease within 15 weeks of providing informed & Solely treated by surgical and radiation therapy. \\
consent. & Receiving complementary care by a professional palliative \\
Scheduled for a first-line anticancer treatment. & care team. \\
& Already participating in another psychosocial study.
\end{tabular}

between the intervention and control groups. ${ }^{42}$ Cluster-randomised trials need thorough sample and cluster size estimations, ${ }^{43}$ which are based on assumptions about the relevant effect size, recruitment potential and intracluster correlation; because reliable information on these parameters is not available, we decided to conduct a pilot study based on a sufficient but feasible sample size. We will use this pilot study to estimate effect sizes and sample size needed for future studies, and to monitor patient safety. ${ }^{44}$ For the evaluation of the RE-AIM dimensions reach and effectiveness, we will mainly apply quantitative methods; for the dimensions adoption and implementation, we will use qualitative methods.

\section{Setting and eligibility criteria}

The study will take place at nine outpatient cancer centres in the German-speaking parts of Switzerland. Cancer centres providing systemic outpatient anticancer treatments (chemotherapy, targeted therapy, immunotherapy- and hormonal therapy) will be eligible. We will exclude outpatient cancer centres where a former version of the SNCP is already implemented. Eligibility criteria for patients are listed in table 1 .

Registered and regularly employed nurses who have worked for at least 1 year in cancer care will provide the SN@P. Physicians with at least 1 year experience in oncology will be involved to assess the acceptance of the $\mathrm{SN} \odot \mathrm{P}$ at an institutional level.

\section{Intervention: SN@P}

The SN@P is a nurse-led intervention to facilitate cancer patients' symptom self-management including semistructured consultations with the involvement of symptom-specific information leaflets (Symptom Navi@ Flyers (SN@Flyers)). We outlined in the nurse-training manual the delivery of the consultation and the use of flyers. The development of the $\mathrm{SN} \odot \mathrm{P}$ was guided by patient education principles considered effective in patients with chronic health conditions, such as building partnership with patients, focusing on patients' needs ${ }^{11} 45$ and self-management strategies. ${ }^{9} 1012$ The development, content and evaluation of the $\mathrm{SN} \odot \mathrm{P}$ are detailed in a separate manuscript (Bana et al, in preparation).

\section{Symptom Navi@ Flyers}

SNCFlyers are written leaflets about 16 commonly occurring symptoms that patients may experience with anticancer treatments (table 2). Each SN@Flyer describes one symptom, guides patients to rate the severity of the symptom (mild, moderate, severe) and provides easy understandable evidence-based recommendations for symptom relief and management. If a patient perceives a symptom to be severe, they are asked to immediately contact the treating outpatient centre. During the development phase of the SNCFlyers, the contents were evaluated by 48 healthcare professionals and patients using the Item Content Validity Index (I-CVI) ${ }^{46}$ achieving an excellent I-CVI of $0.9 .^{47}$ In addition, 10 cancer patients who used the SN@Flyers, confirmed the utility of the recommendations for self-management and the benefit of semistructured nurse-led consultations, assessed with semistructured interviews. ${ }^{48}$

\section{Semistructured nurse-led consultations}

Nurses will provide two semistructured consultations with all patients starting a first-line systemic anticancer treatment, tailored to the patient's treatment protocol and expected side effects. These consultations are structured along six key elements: (1) preparing the semistructured consultation and choosing relevant SNCFlyers, (2) evaluating patient's willingness and motivation for a consultation, (3) providing information on common side effects with the SN@Flyers, (4) introducing symptom self-management, (5) facilitating symptom self-management and (6) documenting the consultation. ${ }^{49}$ Patient's willingness will be assessed by asking his consent for the consultation. The interpretation of patient's motivation will be based on the active participation and being attentive during the conversation. Nurses will have to structure the key elements according to patient's needs, often leading to circular and iterative conversation sequences. ${ }^{11} 12$ While the first semistructured consultation will focus on explaining expected side effects and how to use the SN@Flyers at home, all following consultations will explicitly focus on a patient's individual situation and needs. Nurses may provide additional written information as available at their centres and will decide on whether or not further consultations are needed. The SNCP is an intervention that aims to stimulate patient's self-management of symptoms and complements usual care, which mainly focuses on information provision. Differences between centres regarding information provision might be a bias. To reduce this bias, we will record all additional information material delivered at each centre and report them descriptively (brochures, leaflets). Motivational interviewing techniques (resisting 
Table 2 Available SNCFlyers and timing of semistructured nurse-led consultations

\begin{tabular}{|c|c|}
\hline Available SN@Flyers & Timing of semistructured nurse-led consultations \\
\hline $\begin{array}{l}\text { Leaflets for symptom self-management: } \\
\text { Alopecia. } \\
\text { Anxiety. } \\
\text { Breathlessness. } \\
\text { Diarrhoea. } \\
\text { Emesis and nausea. } \\
\text { Fatigue. } \\
\text { Increased susceptibility: infections and bleeding. } \\
\text { Irradiated skin. } \\
\text { Loss of appetite. } \\
\text { Inflamed oral mucosa. } \\
\text { Obstipation. } \\
\text { Pain. } \\
\text { Peripheral neuropathy. } \\
\text { Sexuality. } \\
\text { Skin alteration: feet and hand. } \\
\text { Comin alterations related to target therapies. } \\
\text { Comentary leaflets: } \\
\text { General information on SN@Flyers. } \\
\text { Information on oxaliplatin. } \\
\text { List of all available SN@Flyers. } \\
\text { Support at home (useful addresses). }\end{array}$ & $\begin{array}{l}\text { First semistructured consultation: } \\
\text { All patients will be provided with the leaflets "General information } \\
\text { onSNCFlyers" and "list of all available SNCFlyers", and } \\
\text { approximately three symptom-specific SN@Flyers based on most } \\
\text { expected side effects in line with planned treatment protocol; this } \\
\text { consultation takes place during the first treatment application at } \\
\text { the outpatient cancer centre. } \\
\text { Second semistructured consultation: } \\
\text { Patients will be provided with complementary SN@Flyers based } \\
\text { on their experienced symptoms and needs. This consultation } \\
\text { takes place during the second treatment application at the } \\
\text { outpatient cancer centre. }\end{array}$ \\
\hline
\end{tabular}

SN@Flyers, Symptom Navi@ Flyers.

the 'righting reflex', understanding patient's motivation, listening with empathy and empowering the patient) will be used to enable patient's active participation during the conversation, to support patient's self-efficacy and to facilitate behavioural changes, if needed. ${ }^{50}$ Patients will be invited to ask for additional SN@Flyers if they desire more information.

\section{Training for nurses}

The two trainers are members of the research team that developed the training courses, hold a master degree in nursing science and are senior lecturers. Nurses will be trained with two standardised training courses (in total 6 hours of training) based on a training manual that has been face validated by a steering committee including two clinical experts for oncology nursing, a nursing manager and two researchers. Details about the content of the trainings are described in table 3. Between the initial and the follow-up training, nurses will practise semistructured consultations using the SN@Flyers according to the initial training and the training manual.

Table 3 Objectives and content of SNOP training courses

\begin{tabular}{|c|c|c|}
\hline Training and duration & Objective & Content \\
\hline Initial training: about 4 hours & Introduce SNOP & $\begin{array}{l}\text { Self-efficacy. } \\
\text { Symptom self-management. } \\
\text { Nurse-patient communication. } \\
\text { Strategies for selecting appropriate SNOFlyers. } \\
\text { How to conduct semistructured consultations } \\
\text { according to the six key elements*. } \\
\text { Motivational interviewing techniques. }\end{array}$ \\
\hline Follow-up training: about 2 hours & Reinforce acquired knowledge/skills & $\begin{array}{l}\text { Answering nurses' questions regarding their } \\
\text { experience with providing the semistructured } \\
\text { consultations embedded in discussions and role } \\
\text { plays }\end{array}$ \\
\hline
\end{tabular}

*Six key elements: (1) preparing the semistructured consultation and choosing relevant SN@Flyers, (2) evaluating patient's willingness and motivation for a consultation, (3) providing information on common side effects with the SNOFlyers, (4) introducing symptom selfmanagement, (5) facilitating symptom self-management and (6) documenting the consultation.

SNOFlyers, Symptom Navi@ Flyers (written information leaflets for patients). 
Additionally, nurses will receive a handbook and pocket cards to facilitate the implementation of semistructured consultations within their daily routines. Pocket cards provide nurses with concrete examples how to guide the communication during the consultations based on motivational interviewing techniques. Nurses will use the pocket cards during consultations. The follow-up training will address nurses' experience with the $\mathrm{SN} \odot \mathrm{P}$, as well as questions and potential challenges that might have occurred during the semistructured consultations.

\section{Control: usual nursing care supporting self-management of symptoms}

Nurses at the control centres will provide symptom management support according to their usual practice. This generally includes providing oral information about expected side effects of treatments, handing out written information as available at the centre (eg, pharmaceutical and Swiss Cancer League brochures) and getting in touch with patients by phone calls, if needed.

\section{Outcomes}

Outcomes will be based on the RE-AIM framework and represent individual and organisational levels. We will assess patient accrual and retention rates (reach); evaluate the impact of the $\mathrm{SN} \odot \mathrm{P}$ on patient-reported outcomes (effectiveness); explore barriers and facilitators at participating centres to deliver the intervention (adoption); evaluate nurses' fidelity to the training manual in routine clinical practice and patients' evaluation on nurses' support for symptom management (implementation)..$^{38}$ Outcomes addressing effectiveness will be based on the theory of symptom self-management (TSSM). ${ }^{751}$ The TSSM addresses five patient-related dimensions: (1) perceived self-efficacy, (2) current symptoms, (3) symptom self-management behaviours, (4) demographic and psychosocial characteristics and (5) functional status (performance outcomes). This framework considers self-efficacy as a mediator between symptom intensity and patients' functional status. An overview of used instruments to assess patient-reported outcomes are provided in table 4, and an overview of all study outcomes, data collection methods and assessment schedule are provided in table 5 .

Reach

Patients' characteristic data will include medical (age, gender, diagnosis, co-morbidities, pharmaceutical information of treatment and Karnovsky index) and sociodemographic information (mother tongue, housing context, highest education degree). Accrual and retention rates of patients' participation in the study will be obtained by recruitment logs completed at each site.

\section{Effectiveness}

The main outcome of interest will be the mean change in symptom interference with daily functions from baseline (BL) (ie, before treatment starts) to 16 weeks after BL. The rationale for the primary outcome is based on a previous study using the TSSM reporting that patients' functional performance increased after nurse-led interventions on symptom self-management support. ${ }^{51}$ Number and type of experienced symptoms depend on cancer type and treatment. ${ }^{52}$ We therefore chose a period of 16 weeks assuming

Table 4 Instruments used to assess patient-reported outcomes

\begin{tabular}{ll}
\hline $\begin{array}{l}\text { Instruments } \\
\text { (RE-AIM dimension) }\end{array}$ & Outcomes \\
\hline $\begin{array}{l}\text { MDASI } \\
\text { (effectiveness) }\end{array}$ & $\begin{array}{l}13 \text { items on symptoms, } \\
\text { and } 6 \text { items on symptom } \\
\text { interference with daily } \\
\text { functions }\end{array}$ \\
$\begin{array}{l}\text { SES6G } \\
\text { (effectiveness) }\end{array}$ & $\begin{array}{l}6 \text { items on patient's perceived } \\
\text { self-efficacy }\end{array}$
\end{tabular}

Scale

11-point Likert-scale, $0=$ not present and $10=$ as bad as you can imagine

10-point Likert-scale, $1=$ not at all confident and 10=totally confident

\section{Validity/reliability}

Developed for cancer setting

German version: Cronbach alpha 0.82 (symptom intensity) and 0.84 (interference) $^{54}$

Developed for chronic conditions, applied in cancer settings German version: construct validity $r=0.578, p<0.001$; internal consistency: Crohnbach alpha $0.93^{55}$

$\begin{array}{lll}\text { LASA Mood Scale } & \text { 1-item: emotional well-being } & \text { Visual Analogue Scale } \\ \text { (effectiveness) } & (100 \mathrm{~mm}), 0=\text { happy } \\ & 100=\text { miserable }\end{array}$

$\begin{array}{lll}\begin{array}{l}\text { PR-CISE } \\ \text { (implementation) }\end{array} & \begin{array}{l}5 \text { items on patient's } \\ \text { experience of nurse-led } \\ \text { supportive care }\end{array} & \text { Yes; somewhat; no } \\ & \end{array}$

LASA, linear analogue self-assessment; MDASI, MD Anderson Symptom Inventory; PR-CISE, patient-reported chemotherapy indicators of symptoms and experiences; RE-AIM, Reach Effectiveness-Adoption Implementation Maintenance; SES6G, self-efficacy for managing chronic disease. 
Table 5 Overview of assessed outcomes based on RE-AIM framework

\section{RE-AIM dimensions}

(Level of evaluation)

Reach

(Individual)

Effectiveness

(Individual)
Patients' characteristics, accrual and retention rates

Symptom interference with daily function (activity and affective subdimensions), symptom severity, overall symptom burden, SES6G, LASA Mood Scale self-efficacy, mood

$\begin{array}{lll}\begin{array}{l}\text { Adoption } \\ \text { (Organisational) }\end{array} & \begin{array}{l}\text { Characteristics of participating centre and } \\ \text { staff, usual support for SSM, facilitators } \\ \text { and barriers for SNOP adoption }\end{array} & \begin{array}{l}\text { Focus group interviews (1), } \\ \text { electronic questionnaires }\end{array} \\ \begin{array}{ll}\text { Implementation } \\ \text { (Organisational) }\end{array} & \begin{array}{l}\text { (1) Acceptance and appropriateness of } \\ \text { nurses' trainings: training content, nurses' } \\ \text { confidence to integrate SNOP into practice, } \\ \text { work-related factors with implementing } \\ \text { SNOP }\end{array} & \begin{array}{l}\text { Paper and pencil } \\ \text { questionnaire, }\end{array} \\ & \text { WoC scale }\end{array}$

(2) Nurses' and physicians' acceptance of SNOP

(3) Nurses' fidelity to training manual: key elements applied, patients' complaints and goals, number and topic of delivered SNOFyers, additionally delivered information leaflets

(4) Patients' safety

(5) Resources: time needed for trainings, preparing and providing nurse-led consultations, documentation

(6) Quality of nursing care estimated by patients

Maintenance Not applied

\section{Data collection methods Assessment schedule}

Medical records, recruitment Study start: patients' logs at each centre recruitment and enrolment

Paper and pencil

Paper and pencil Four measurements over 16 weeks: from $B L$ to $t 3$

Study start: before patient recruitment starts

After first and second training

Focus group interviews (2) and telephone interviews

After patients completed all questionnaires (last patient out of study)

Electronic questionnaires

Observations

After every semistructured consultations

Focus group interviews (1): takes place before patient recruitment starts at the centre; Focus group interviews (2): takes place after last patient is out of study.

BL, baseline; MDASI, MD Anderson Symptom Inventory; PR-CISE, patient-reported chemotherapy indicators of symptoms and experiences; RE-AIM, Reach Effectiveness-Adoption Implementation Maintenance; SES6G, Self-Efficacy for Managing Chronic Disease Questionnaire; SSM: Symptom Self-Management; SN@P: Symptom Navi@ Program; SN@Flyers: Symptom Navi@ Flyers (written information leaflets for patients); Work-SoC, Work-related Sense of Coherence scale.

that most patients are still under treatment. We also assume that the SN@P might affect patient's estimation on symptom interference over this period, because patient's positive attitude for self-management has been shown to be associated with increased physical, emotional and functional well-being over 6 months. ${ }^{53}$ Other outcomes will include mean changes in symptom intensity, perceived self-efficacy and quality of nursing care assessed four times over a period of 16 weeks. The following outcomes to assess effectiveness will be used:

- Symptoms severity, their interference with daily functioning and symptom burden will be assessed by the validated German version of the $M D$ Anderson Symptom Inventory (MDASI) ${ }^{54}$ The MDASI contains two dimensions: (1) the severity of thirteen common symptoms and (2) the interference of these symptoms with daily function on an activity and an affective subdimension. The dimensions symptom severity and symptom interference summarise an overall symptom burden score.

- Perceived self-efficacy will be assessed with the validated German version of the Self-Efficacy for Managing Chronic Disease Scale (SES6G).$^{55}$ We added an item asking, 'how confident do you feel that you can manage your symptoms to be able to do things you would like to do?' This general question on perceived self-efficacy complements the more specific items of the SES6G (eg, self-efficacy for managing fatigue or pain).

Depressive moods might affect a person's belief to accomplish a desired behaviour or to achieve a target outcome (self-efficacy). ${ }^{56}$ To control for the emotional state of the patients, we added a one-item Visual Analogue Scale on mood asking 'how do you rate your mood during the last two weeks?' ${ }^{57}$ 


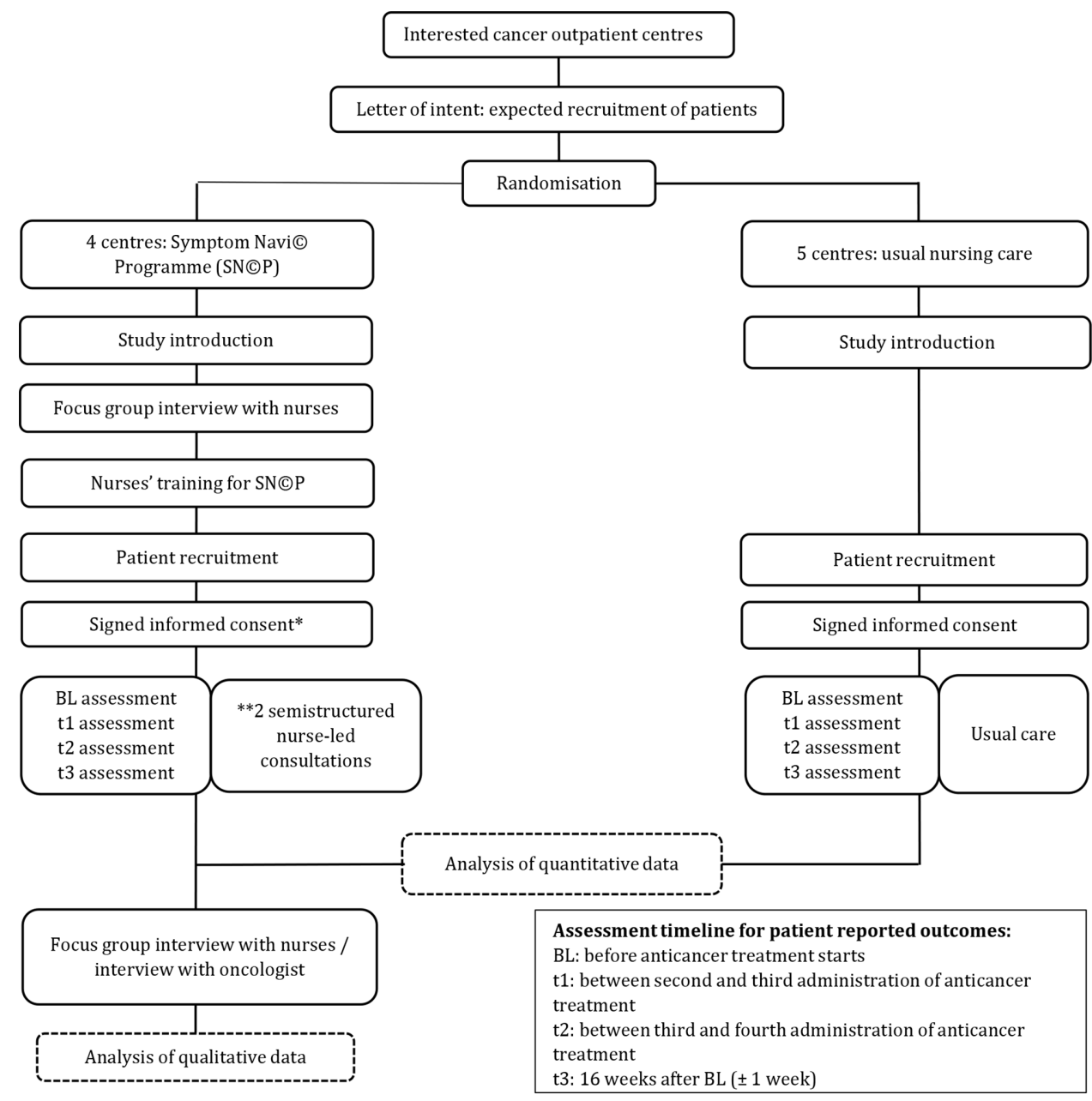

Figure 1 Study flowchart for the Symptom Navi@ Pilot Study with included patient timeline. *Every patient enrolled for the pilot study will start with SNCP and will be followed by two semistructured nurse-led consultations. ${ }^{* \star}$ Semistructured nurse-led consultations take place during first and second scheduled treatment application at the outpatient centres of the intervention arm. BL, baseline.

\section{Adoption}

We will assess the characteristics of participating outpatient cancer centres and nurses (ie, specialised cancer centre, nurses' formation, number of employed nurses and oncologists at each intervention centre, average number of delivered anticancer treatments per day, number of treated patients at the centre per year, information leaflets usually delivered to patients).

We will conduct a first focus group interview with nurses before they will be trained for the SN@P to learn about the current symptom self-management support and handling of written information at each intervention centre. A second focus group interview (after last patient is out of study at the centre) will be conducted with those nurses who provided the intervention to assess perceived barriers and facilitators (eg, work-related factors, available resources) for adopting the $\mathrm{SN} \odot \mathrm{P}$ within daily routines (figure 1 ).

Interview guidelines for semistructured focus groups will be based on Morgan. ${ }^{58}$

\section{Implementation}

To evaluate the success of the implementation we will assess:
1. Acceptance and appropriateness of the nurse-training course by using a 5-item paper and pencil questionnaire based on the training manual. We developed five questions regarding content and acceptability using 7-point Likert scales rated from 1 (not at all) to 7 (greatest possible). Two open-ended questions for narrative feedback on both training courses complement the Likert scales. To assess potentially influencing work-related factors for implementing the SN@P into practice, we added the Work-related Sense of Coherence (Work-SoC) scale. ${ }^{59}$ The Work-SoC scale is a 9-item validated screening instrument for assessing employees' perceived quality of work situation on three subscales: comprehensibility, manageability and meaningfulness. ${ }^{59}$

2. Acceptance and feasibility of the $\mathrm{SN} \odot \mathrm{P}$ within daily routines will be explored using focus group interviews with nurses and a telephone interview with one oncologist from each of the intervention centres. Interviews with oncologists were included to represent the institutions voice regarding acceptance and feasibility of the $\mathrm{SN} \odot \mathrm{P}$ within daily routines. Focus group and tele- 
phone interviews will be directed by semistructured interview guidelines. ${ }^{58} 60$ Topics addressed in both interviews will focus on symptom self-management support based on the frameworks of Howell et $a l^{13}$ and Schofield and Chambers. ${ }^{15}$

3. Nurses report on fidelity to the training manual by using electronic questionnaires. These questionnaires were developed based on the six key elements of semistructured consultations. Sixteen questions are in dichotomous format (yes-no); three text fields are added for reporting patients' complaints, their goals regarding symptom self-management behaviours, and observed 'unsafe' behaviour of patients. We consider as unsafe behaviour, for example, a delayed reaction of a patient despite severe symptoms such as fever with neutropenia or exacerbated diarrhoea. In addition, a study team member will observe two semistructured consultations at each centre by using the above-mentioned questionnaire in printed format to record observed behaviour of nurses.

4. Patients' safety will be also explored with focus group interviews with nurses and telephone interviews with oncologists. Serious adverse events will be assessed electronically according to authority guidelines. ${ }^{6162}$

5 . Resources needed to implement the SN@P at the centres will be assessed based on training duration and number of participating nurses documented on training logs. Nurses will assess electronically time needed for semistructured consultations including preparation and documentation of consultations.

6. Quality of nursing care evaluated by patients will assess five concerns: do nurses ask patients about symptoms, provide useful information, and/or practical advice to manage symptoms, are they aware of patient's symptom severity, and whether patients feel confident to manage symptoms. The patient-reported chemotherapy indicators of symptoms and experiences (PR-CISE) ${ }^{28}$ is a quality measure for outpatient chemotherapy settings. We translated five items of the original PR-CISE questionnaire following a forward and backward translation process based on a translation and cultural adaptation guideline. ${ }^{63}$ The translation was reviewed by two nursing experts and pilot-tested with 10 cancer patients from an outpatient cancer centre that will not participate in this study.

\section{Sample size and randomisation}

We aim to include a total of 140 patients in 9 clusterswith $\sim 70$ patients to be included in both the intervention and the control group, and about 10-20 patients in each cluster (at each centre).

Due to the lack of data on the expected magnitude of effect of SN@P on outcomes, we did not formally calculate a sample size, ${ }^{44}$ but rather estimated the power for the expected sample size. Assuming an intraclass correlation of 0.05 , a type I error rate of $5 \%$ and an equal distribution of the patients among the clusters, a total sample size of 135 patients (ie, 9 clusters with 15 patients) would allow a detection of an effect size of $0.5,0.75$ and 1 with powers of about $60 \%, 91 \%$ and $99 \%$, respectively, based on a two-sample comparison of means in a cluster-randomised design.

The Clinical Trial Unit of the University of Bern (CTU Bern) will execute the randomisation at the level of cancer outpatient centres. Randomisation will be stratified by the expected recruitment potential (fast vs slow recruiters) and will be based on randomly permuted blocks with a block size of two to minimise potential imbalances within the small number of included clusters. We assume that nurses are more familiar with treatment protocols at faster recruiting centres, and therefore might also be more experienced with supporting patients during anticancer treatments. Stratification will also help with balancing the number of patients between treatment groups since cluster size depends on the recruitment potential. We will not implement allocation concealment or blinding procedures.

\section{Data collection and management}

A data capturing system (secuTrial) will be set up for data entry at CTU Bern for all quantitative data. Nurses and physicians involved with the study procedures will have a personal login to secuTrial for data recording. A dedicated nurse and a principal investigator (an oncologist who has worked for at least 1 year at the centre) at each centre will be responsible for identifying eligible patients for study inclusion and informing patients orally and in written format about the study.

The study procedure is summarised in a participants' flowchart (see figure 1). The BL assessment will take place before patients start their first treatment application at the outpatient cancer centre. Two further assessments will take place between subsequent treatment applications (t1 between second and third, t2 between third and fourth treatment application) when the patient is at home. This takes into account the variety of treatment protocols with different administration schedules. The last assessment (t3) will again be completed by the patient at home, 16 weeks ( \pm 1 week) after the BL assessment.

Nurses will hand over questionnaires and prestamped addressed envelopes to patients and inform them when they should fill in the questionnaire at home. Returned questionnaires will be entered centrally into secuTrial by a study team member. After every semistructured consultation with a patient, nurses will complete an electronic questionnaire assessing their fidelity to the training manual, and patient's complaints and goals for symptom self-management as discussed during the consultation.

All focus group interviews will be audio recorded and transcribed. After patient recruitment is completed at an intervention centre, a telephone interview with one oncologist will be conducted. Data management of qualitative data will be based on excel sheets and logbooks, if applicable.

Patient recruitment started in November 2017. We expect the last patient to complete the study by the end of April 2019 and to complete the qualitative data assessment by the end of June 2019. 


\section{Analysis}

The null hypothesis, that there is no difference in the changes of symptom interference score of the MDASI between intervention and control group, will be tested against a two-sided alternative. We will perform a primary analysis on the intention-to-treat population (ie, analysing all patients according to the intervention they were assigned to at randomisation) and a secondary analysis on the perprotocol population (ie, excluding patients who were not treated according to protocol). All effectiveness outcomes will be analysed using linear or generalised linear mixed-effects models. BL measurement, treatment group, time point (ie, t1, t2 or t3) and the interaction of group and time point will be included as fixed covariates, cluster and patient as nested random effects. We will present all results using an effect measure with a two-sided $95 \%$ CI and a $\mathrm{p}$ value. In a sensitivity analysis, we will adjust the model for potential confounders, that is, patient, nurse or cluster characteristics that show imbalances at BL.

All other outcomes will be analysed descriptively. A statistical analysis plan with a detailed description of data preparation and analysis will be written in collaboration with a statistician before completion of recruitment. Quantitative analysis will be performed in collaboration with the CTU in Bern using an appropriate statistical software (eg, R 3.6.0 or STATA 14.2).

Transcripts of focus group and individual interviews, as well as narrative information from the questionnaires on fidelity including patients' goals for symptom self-management will be analysed by thematic analysis. ${ }^{64}$ Thematic analysis is a six-phase approach to identify patterns (themes): (1) familiarising with data, (2) generating initial codes, (3) searching for themes, (4) reviewing themes, (5) defining and naming themes and (6) producing the report. ${ }^{64}$ Transcripts will be coded independently by two members of the research team. A third member will be involved to discuss discordances between the two coders until consensus is reached.

\section{Patient and public involvement}

We did not involve patients or public for developing this pilot study. Results of the study will be presented at each participating cancer outpatient centre. An assessment of patients' burden of the intervention was not included in the pilot study based on previous evaluation of the intervention from patients' perspective confirming that the SNOP did not cause burden for patients.

\section{ETHICS AND DISSEMINATION}

This pilot study has been reviewed by four Swiss Ethics Committees and approved by the Swiss Ethic Committee in Bern (KEK-BE: 2017-00020), and will be conducted in accordance to the Declaration of Helsinki ${ }^{65}$ and the International Council for Harmonisation of Technical Requirements for Pharmaceuticals for Human Use/ Good Clinical Practice (ICH-GCP) guidelines. ${ }^{66}$ Any modification of the protocol will be submitted to and approved by the leading Ethic Committee in Bern. Patients will sign a written informed consent form (online supplementary file 1) before being included in the study. Signed informed consent forms and patient enrolment logs will be stored at the outpatient cancer centres. All data will be anonymised when presented at scientific meetings or published. Serious adverse events will be assessed by local principal investigators and evaluated according standard serious adverse reporting procedures ${ }^{67}$ We registered this pilot study at ClinicalTrials.gov and at the Swiss National Clinical Trials Portal (SNCTP): SNCTP000002381.

Results of this study will be disseminated at national and international conferences and published in peer-reviewed journals with a preference of open access journals. Nurses at the control group centres will be trained on the SN@P to implement it at their centre after pilot study completion and confirmation that the SN@P can be considered to be safe. If the safety of the SN@P will be confirmed with this study, we plan to collaborate with the Swiss Cancer League for broader dissemination of the SN@P in Switzerland. Supporting self-management strategies of cancer patients is an explicit aim of the Swiss National Strategy against Cancer. ${ }^{686}$

\section{DISCUSSION}

The Symptom Navi@ Pilot Study aims to evaluate the implementation of the $\mathrm{SN} \odot \mathrm{P}$ within daily routines. We will evaluate preliminary effectiveness and safety of the intervention on patient-reported interference of symptoms with daily functions, symptom intensity and burden, perceived self-efficacy and quality of nursing care. The results may contribute to greater insight into the mediating role of self-efficacy for self-management of symptoms. ${ }^{51}$ We expect the SN@P to enhance nurse-led support for cancer patients in the outpatient setting. Estimated effect sizes will serve for effect and sample size calculations for a fully powered cluster randomised controlled clinical trial.

Successful implementation of complex interventions depends on providing the intervention as intended, but also on contextual factors. ${ }^{32}{ }^{39}$ To meet these challenges, we have designed a study based on the RE-AIM framework using a cluster-randomised design complemented with qualitative methods. ${ }^{70}$ The SN@P has been thoroughly developed ${ }^{49}$ and patients confirmed that they could improve their self-management behaviours by using SN@Flyers. ${ }^{71}$ Therefore, the SN@P is a promising nurse-led intervention to support patients' symptom self-management and enrich current usual care practices in the outpatient cancer setting, but its implementation and effectiveness need to be investigated.

\section{Author affiliations}

${ }^{1}$ HedS-FR School of Health Sciences, University of Applied Science and Arts Western Switzerland, Fribourg, Switzerland

${ }^{2}$ IUFRS Institut de formation et de recherche en soins, Université de Lausanne Faculté de biologie et médecine, Lausanne, Switzerland

${ }^{3}$ Quality ofLife Office, International Breast Cancer Study Group, Bern, Switzerland

${ }^{4}$ Fachentwicklung Pflege, Lindenhofgruppe, Bern, Switzerland 
${ }^{5}$ Department of Practice Development in Nursing, Solothurner Spitaler AG, Solothurn, Switzerland

${ }^{6}$ FHNW School of Applied Psychology, University of Applied Sciences and Arts Northwestern Switzerland, Olten, Switzerland

${ }^{7}$ CTU, Universität Bern, Bern, Switzerland

${ }^{8}$ Departement of Oncology, CHUV, Lausanne, Switzerland

Acknowledgements We acknowledge Dr rer. medic. Patrick Jahn and Dr sc. (ETH) Susanne Hoffmann for their advise and support regarding study design and the evaluation of nurses' training. Further, we acknowledge all participating outpatient centres with local investigators and nurses who support this pilot study: Gynäkologisches Tumorzentrum, Universitätsspital Basel: Professor Dr med. Viola A Heinzelmann, Veronica Fasanella, Verena Fluri, Fabienne Hess, Eveline Schönau, Jasmina Kljajic, Franziska Schmidle, Helena Strebel, Shqipc Bucaliu ; Hôpital fribourgeois-Meyriez-Murten: Professor Dr med. D Betticher, Dr med. Vérène Dougoud Chauvin, Priska Koch, Claudia Schmid, Sophie Renevey; Kantonsspital Aarau: Dr med. Nathan Cantoni, Thomas Seger, Sina Brugger, Fatima Dos Santos Oliveira, Thomas Widmer, Stefan Büschl, Therese Grädel, Ursula Neumann, Denise Gloor; Kantonsspital Graubünden: Dr med. Michael Schwitter, Barbara Stoffel, Sabrina Zortea, Anja Cathomas, Gabriela Manetsch; Brustzentrum Bern, Engeried Spital: Professor Dr med. Markus Borner, Dr med. Michele Ciriolo, Dr. med. Katharina Buser, Chantal Schneider, Isabelle Steiner, Anja Blunschi, Ditte Immoberdorf, Claudia Vögeli, Madeleine Dittens, Dr med. Claudia Gübelin; Rundum Onkologie am Bahnhofpark Sargans: Dr med. Stefan Greuter, Renata Marthy, Michela Winter, Diana Malin; Solothurner Spitäler AG—Kantonsspital Olten/ Bürgerspital Solothurn: Dr med. Thomas Egger, Dr med. Walter Mingrone, Dr med. Andreas Barth, Dr med. Simone Farese, Dr med. Phillipe Von Burg, Dr med. Grit Richartz, Dr med. Sybille Wyss, Dr med. Martin Kälin, Ernst Näf, Kathrin Schnyder, Marlies Bogaert, Ruth Jordi, Anita Sidler, Marina Affolter; Spital STS AG—Thun: Dr med. Jean-Marc Lüthi, Sandra Knettenmann, Nadja Rubin, Trudy Kuhn, Christine Kuhn, Francine Rieder Nicolet, Manuel Schnegg, Verena Flügel, Sadiku Fitore, Thorsten Dürmüller; Tumor- und Brustzentrum ZeTuP Rapperswil: Dr med. Rudolf Morant, Dr med. Iris Müller-Käser, Dr med. Daniel Koychev, Lisa Haefliger, Isabel Carrard, Rebecca Biber, Susi Brunner-Heller.

Contributors MB: SNOP development, study design and conduct, manuscript writing. KR: study conduct, manuscript writing. SK-S and EN: SNOP development, study design and conduct, manuscript review. SZ-F: SNOP development, study design, manuscript review. TM and SP: study design, manuscript review. LB and FR: study design and statistical support, manuscript review. ME: SNOP development, study design and conduct, obtained funding, manuscript writing.

Funding This study is funded by: University of Applied Sciences and Arts Western Switzerland, School of Health Sciences Fribourg, Switzerland; Institute of Higher Education and Research in Health Care, Faculty of Biology and Medicine, University of Lausanne, Switzerland; Department of Oncology, Centre Hospitalier Universitaire Vaudois (CHUV), Lausanne, Switzerland; Hospital Group Lindenhof, Bern,

Switzerland; Swiss Cancer League, Bern, Switzerland; Dr Hans Altschüler Stiftung, St Gallen, Switzerland.

Disclaimer The Swiss Cancer League and Dr Hans Altschüler Stiftung provided financial support for reimbursement of participating cancer outpatient centres and for the Clinical Trial Unit. None of these bodies were involved in the study design or writing the manuscript, nor will they be involved in analysis and interpretation of the results of this pilot study.

Competing interests SP has received education grants, provided consultation, attended advisory boards and/or provided lectures for: Abbvie, Amgen, AstraZeneca, Bayer, Biocartis, Boehringerlngelheim, Bristol-Myers Squibb, Clovis, Daiichi Sankyo, Debiopharm, Eli Lilly, F Hoffmann-La Roche, Foundation Medicine, Illumina, Janssen, Merck Sharp and Dohme, Merck Serono, Merrimack, Novartis, Pharma Mar, Pfizer, Regeneron, Sanofi, Seattle Genetics and Takeda , from whom she has received honoraria. ME received education grants, provided consultation, attended advisory boards and/or provided lectures for: Vifor, Roche, and Bristol-Myers Squibb. All other authors have no competing interests to declare.

Provenance and peer review Not commissioned; externally peer reviewed.

Open access This is an open access article distributed in accordance with the Creative Commons Attribution Non Commercial (CC BY-NC 4.0) license, which permits others to distribute, remix, adapt, build upon this work non-commercially, and license their derivative works on different terms, provided the original work is properly cited, appropriate credit is given, any changes made indicated, and the use is non-commercial. See: http://creativecommons.org/licenses/by-nc/4.0/.

\section{REFERENCES}

1. Arndt V, Feller A, Hauri D, et al. Schweizerischer Krebsbericht 2015, Stand und Entwicklungen. Neuchâtel: Bundesamt für Statistik (BFS), Nationales Institut für Krebsepidemiologie und -registrierung (NICER), Schweizer Kinderkrebsregister, 2016 https://www.bfs.admin.ch/bfs/ de/home/statistiken/kataloge-datenbanken/medienmitteilungen. assetdetail.40064.html.

2. Brédart A, Kop JL, Efficace F, et al. Quality of care in the oncology outpatient setting from patients' perspective: a systematic review of questionnaires' content and psychometric performance. Psychooncology 2015;24:382-94.

3. Deshields TL, Potter P, Olsen S, et al. The persistence of symptom burden: symptom experience and quality of life of cancer patients across one year. Support Care Cancer 2014;22:1089-96.

4. Cleeland CS, Zhao F, Chang VT, et al. The symptom burden of cancer: Evidence for a core set of cancer-related and treatmentrelated symptoms from the Eastern Cooperative Oncology Group Symptom Outcomes and Practice Patterns study. Cancer 2013;119:4333-40.

5. Giesinger JM, Wintner LM, Zabernigg A, et al. Assessing quality of life on the day of chemotherapy administration underestimates patients' true symptom burden. BMC Cancer 2014;14:758.

6. Coolbrandt A, Dierckx de Casterlé B, Wildiers $\mathrm{H}$, et al. Dealing with chemotherapy-related symptoms at home: a qualitative study in adult patients with cancer. Eur J Cancer Care 2016;25:79-92.

7. Hoffman AJ. Enhancing self-efficacy for optimized patient outcomes through the theory of symptom self-management. Cancer Nurs 2013;36:E16-E26.

8. Barlow J, Wright C, Sheasby J, et al. Self-management approaches for people with chronic conditions: a review. Patient Educ Couns 2002;48:177-87.

9. Lorig KR, Holman H. Self-management education: history, definition, outcomes, and mechanisms. Ann Behav Med 2003;26:1-7.

10. Bodenheimer $\mathrm{T}$, Lorig $\mathrm{K}$, Holman $\mathrm{H}$, et al. Patient self-management of chronic disease in primary care. JAMA 2002;288:2469-75.

11. Lorig KR. Patient education. A practical approach. 3rd edn. Thousand Oaks: Sage Publications, 2001.

12. McCorkle R, Ercolano E, Lazenby M, et al. Self-management: Enabling and empowering patients living with cancer as a chronic illness. CA Cancer J Clin 2011;61:50-62.

13. Howell D, Harth T, Brown J, et al. Self-management education interventions for patients with cancer: a systematic review. Support Care Cancer 2017;25:1323-55.

14. Bandura A. Self-efficacy. The exercise of control. 1st edn. New York: W.H. Freeman \& Co Ltd, 1997.

15. Schofield P, Chambers S. Effective, clinically feasible and sustainable: key design features of psycho-educational and supportive care interventions to promote individualised selfmanagement in cancer care. Acta Oncol 2015;54:805-12.

16. Bayly J, Wakefield D, Hepgul N, et al. Changing health behaviour with rehabilitation in thoracic cancer: A systematic review and synthesis. Psychooncology 2018;27:1675-94.

17. Kim SH, Kim K, Mayer DK. Self-management intervention for adult cancer survivors after treatment: a systematic review and metaanalysis. Oncol Nurs Forum 2017;44:719-28.

18. Rueda JR, Solà I, Pascual A, et al. Non-invasive interventions for improving well-being and quality of life in patients with lung cancer Cochrane Database Syst Rev 2011;9:CD004282.

19. Scott DA, Mills M, Black A, et al. Multidimensional rehabilitation programmes for adult cancer survivors. Cochrane Database Syst Rev 2013;3:CD007730.

20. Faury S, Koleck M, Foucaud J, et al. Patient education interventions for colorectal cancer patients with stoma: A systematic review. Patient Educ Couns 2017;100:1807-19.

21. Boland L, Bennett K, Connolly D. Self-management interventions for cancer survivors: a systematic review. Support Care Cancer 2018;26:1585-95.

22. Cheng KKF, Lim YTE, Koh ZM, et al. Home-based multidimensional survivorship programmes for breast cancer survivors. Cochrane Database Syst Rev 2017;8:CD011152.

23. Hammer MJ, Ercolano EA, Wright F, et al. Self-management for adult patients with cancer: an integrative review. Cancer Nurs 2015;38:E10-E26.

24. Cockle-Hearne J, Faithfull S. Self-management for men surviving prostate cancer: a review of behavioural and psychosocial interventions to understand what strategies can work, for whom and in what circumstances. Psychooncology 2010;19:909-22.

25. Hurtado-de-Mendoza A, Cabling ML, Lobo T, et al. Behavioral Interventions to Enhance Adherence to Hormone Therapy in Breast Cancer Survivors: A Systematic Literature Review. Clin Breast Cancer 2016;16:247-55. 
26. Kuo CY, Liang SY, Tsay SL, et al. Symptom management tasks and behaviors related to chemotherapy in Taiwanese outpatients with breast cancer. Eur J Oncol Nurs 2015;19:654-9.

27. Olver I, Carey M, Boyes A, et al. The timeliness of patients reporting the side effects of chemotherapy. Support Care Cancer 2018;26:3579-86.

28. Armes J, Wagland R, Finnegan-John J, et al. Development and testing of the patient-reported chemotherapy indicators of symptoms and experience: patient-reported outcome and process indicators sensitive to the quality of nursing care in ambulatory chemotherapy settings. Cancer Nurs 2014;37:E52-60.

29. Wagland R, Richardson A, Armes J, et al. Treatment-related problems experienced by cancer patients undergoing chemotherapy: a scoping review. Eur J Cancer Care 2015;24:605-17.

30. Coolbrandt A, Wildiers $\mathrm{H}$, Laenen $\mathrm{A}$, et al. A nursing intervention for reducing symptom burden during chemotherapy. Oncol Nurs Forum 2018;45:115-28.

31. Coolbrandt A, Wildiers $\mathrm{H}$, Aertgeerts B, et al. Characteristics and effectiveness of complex nursing interventions aimed at reducing symptom burden in adult patients treated with chemotherapy: a systematic review of randomized controlled trials. Int J Nurs Stud 2014;51:495-510.

32. Craig P, Dieppe P, Macintyre S, et al. Developing and evaluating complex interventions: the new Medical Research Council guidance. BMJ 2008;337:a1655

33. Damschroder LJ, Aron DC, Keith RE, et al. Fostering implementation of health services research findings into practice: a consolidated framework for advancing implementation science. Implement Sci 2009;4:50.

34. Grol R, Grimshaw J. From best evidence to best practice: effective implementation of change in patients' care. Lancet 2003;362:1225-30.

35. Serena A, Griesser A-C, Débarge P, et al. Unmet supportive care needs of lung cancer patients during the chemotherapy phase: A descriptive study. Schweizer Krebsbulletin 2012;2:118-22.

36. Dubey C, De Maria J, Hoeppli C, et al. Resilience and unmet supportive care needs in patients with cancer during early treatment: A descriptive study. Eur J Oncol Nurs 2015;19:582-8.

37. Mendel P, Meredith LS, Schoenbaum M, et al. Interventions in organizational and community context: a framework for building evidence on dissemination and implementation in health services research. Adm Policy Ment Health 2008;35:21-37.

38. Glasgow RE, Vogt TM, Boles SM. Evaluating the public health impact of health promotion interventions: the RE-AIM framework. Am J Public Health 1999;89:1322-7.

39. Glasgow RE, Chambers D. Developing robust, sustainable, implementation systems using rigorous, rapid and relevant science. Clin Trans/ Sci 2012;5:48-55.

40. Glasgow RE, McKay HG, Piette JD, et al. The RE-AIM framework for evaluating interventions: what can it tell us about approaches to chronic illness management? Patient Educ Couns 2001;44:119-27.

41. White LL, Cohen MZ, Berger AM, et al. Self-efficacy for management of symptoms and symptom distress in adults with cancer: an integrative review. Oncol Nurs Forum 2019;46:113-28.

42. Campbell MK, Piaggio G, Elbourne DR, et al. CONSORT 2010 statement: extension to cluster randomised trials. BMJ 2012;:e5661.

43. Hemming $\mathrm{K}$, Eldridge $\mathrm{S}$, Forbes $\mathrm{G}$, et al. How to design efficient cluster randomised trials. BMJ 2017;358:j3064.

44. Thabane L, Ma J, Chu R, et al. A tutorial on pilot studies: the what, why and how. BMC Med Res Methodol 2010:10:1.

45. Rankin SH, Stallings KD, London F. Patient education in health and illness. 5th edn. Lippincott Williams \& Wilkins: Philadelphia PA, 2005.

46. Polit DF, Beck CT. The content validity index: are you sure you know what's being reported? Critique and recommendations. Res Nurs Health 2006;29:489-97.

47. Sailer Schramm M, Kropf-Staub S, Näf E, et al. Is the content of the Symptom Navi valid to support symptom self-management of cancer patients? EONS 10, European Oncology Nursing Society, 17-18 October; Dublin, Ireland. 2016.

48. Kropf-Staub S, Sailer Schramm M, Preusse-Bleuer B, et al. Flyer Symptom Navi zur Unterstützung des Selbstmanagements von Symptomen bei Krebs - Evaluation von Anwendbarkeit und Verständlichkeit. Pflege 2017;30:151-60.

49. Kropf-Staub S, Sailer Schramm M, Zürcher S, et al. Symptom Navi Programm - Entwicklung 2011 - 2015. Onkologische Pflege $2017: 7 \cdot 21-7$
50. Rollnick S, Miller WR, Butler CC. Motivational interviewing in health care, helping patients change behavior. New York: The Guilford Press, 2008:203.

51. Hoffman AJ, Brintnall RA, Given BA, et al. Using perceived selfefficacy to improve fatigue and fatigability in postsurgical lung cancer patients: a pilot randomized controlled trial. Cancer Nurs 2017;40:1-12.

52. Fox $\mathrm{P}$, Darley A, Furlong $\mathrm{E}$, et al. The assessment and management of chemotherapy-related toxicities in patients with breast cancer, colorectal cancer, and Hodgkin's and non-Hodgkin's lymphomas: A scoping review. Eur J Oncol Nurs 2017;26:63-82.

53. Papadopoulou C, Kotronoulas G, Schneider A, et al. PatientReported Self-Efficacy, Anxiety, and Health-Related Quality of Life During Chemotherapy: Results From a Longitudinal Study. Oncol Nurs Forum 2017:44:127-36.

54. Schmidt $\mathrm{H}$, Cleeland CS, Bauer A, et al. Symptom burden of cancer patients: validation of the German M. D. Anderson symptom inventory: a cross-sectional multicenter study. J Pain Symptom Manage 2015;49:117-25.

55. Freund T, Gensichen J, Goetz K, et al. Evaluating self-efficacy for managing chronic disease: psychometric properties of the six-item Self-Efficacy Scale in Germany. J Eval Clin Pract 2013;19:39-43.

56. Shim E-J, Lee JW, Min YH. Does depression decrease the moderating effect of self-efficacy in the relationship between illness perception and fear of progression in breast cancer? Psychooncology 2018;27:539-47.

57. Hürny $\mathrm{C}$, Bernhard J, Coates $\mathrm{A}$, et al. Responsiveness of a singleitem indicator versus a multi-item scale: assessment of emotional well-being in an international adjuvant breast cancer trial. Med Care 1996;34:234-48

58. Morgan DL. Focus groups as qualitative research. Thousand Oaks, California: Sage Publications, 1997.

59. Vogt K, Jenny GJ, Bauer GF. Comprehensibility, manageability and meaningfulness at work: Construct validity of a scale measuring work-related sense of coherence. SA Journal of Industrial Psychology 2013;39.

60. Creswell JW. Data Collection. Qualitative Inquiry \& Research Design. 3rd edn. Thousand Oaks, California: Sage Publications, 2013:145-78.

61. Humanforschungsgesetz HFG. 2011 https://www.admin.ch/opc/de/ classified-compilation/20061313/index.html.

62. Verordnung über klinische Versuche in der Humanforschung. 2013 https://www.admin.ch/opc/de/classified-compilation/20121176/ index.html.

63. Wild D, Grove A, Martin M, et al. Principles of good practice for the translation and cultural adaptation process for Patient-Reported Outcomes (PRO) Measures: report of the ispor task force for translation and cultural adaptation. Value in Health 2005;8:94-104.

64. Braun V, Clarke V. Using thematic analysis in psychology. Qual Res Psychol 2006;3:77-101.

65. World Medical Association (WMA). Declaration of Helsinki, Version October 2013: World Medical Association, 2013. https://www.wma. net/publications/world-medical-journal/wmj201306/.

66. Harmonisation for Better Health (ICH). General considerations for clinical trials. http://www.ich.org/products/guidelines/efficacy/article/ efficacy-guidelines.html1997

67. International Conference on Harmonisation (ICH) Expert Working Group. Guideline for good clinical practice E6(R1). http://www.ich. org/products/guidelines/efficacy/article/efficacy-guidelines.html1996

68. Gasser C, Röthlisberger M, Kramis K, et al. Weiterführung Nationale Strategie gegen Krebs 2017 - 2020. Bern, 2013 https://www.bag. admin.ch/bag/de/home/strategie-und-politik/nationale-gesundheitss trategien/nationale-strategie-gegen-krebs-2014-2017.html.

69. Kramis K, Ruckstuhl B, Wyler M. Nationale Strategie gegen Krebs 2014 - 2017. Bern: Departement des Inneren BfG, 2013 https://www. bag.admin.ch/bag/de/home/strategie-und-politik/nationale-gesu ndheitsstrategien/nationale-strategie-gegen-krebs-2014-2017.html.

70. Gaglio B, Shoup JA, Glasgow RE. The RE-AIM framework: a systematic review of use over time. Am J Public Health 2013;103:e38-e46.

71. Kropf-Staub S, Eicher M, Haselbeck J, et al. Welche Erfahrungen machen an Krebs erkrankte Menschen mit einem Symptommanagement - Patientenedukationstool? Eine thematische Analyse Berner Fachhochschule 2016 unpublished Master Thesis.

72. Singer S, Danker H, Dietz A, et al. Screening for mental disorders in laryngeal cancer patients: a comparison of 6 methods. Psychooncology 2008;17:280-6. 